Supplementary Information for the Manuscript

\title{
Metal Azolate/Carboxylate Frameworks as Catalysts in Oxidative and C-C Coupling Reactions
}

Aurel Tăbăcaru, Nertil Xhaferaj, Luísa M.D.R.S. Martins, ${ }^{*}$ Elisabete C.B.A. Alegria, Rogério S. Chay, Carlotta Giacobbe, Konstantin V. Domasevitch, Armando J.L. Pombeiro, Simona Galli, ${ }^{*}$ Claudio Pettinari ${ }^{*}$ 


\section{S.1 Crystal Structure Analysis}
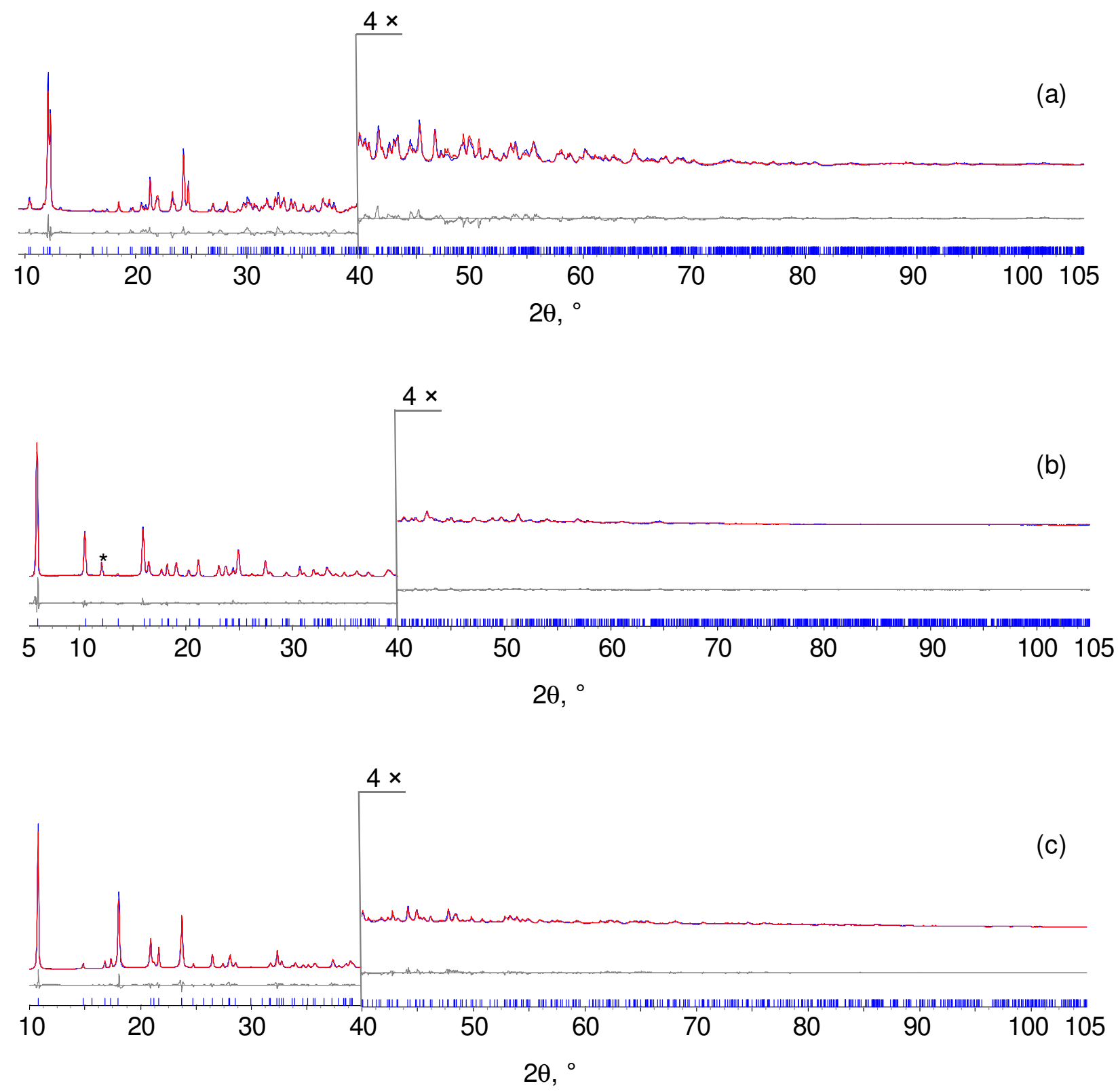

Figure S1: Graphical results of the final Rietveld refinements carried out on: (a) Cd-dmpzc, (b) Pd-dmpzc, and (c) Cu-dmpzc, in terms of experimental, calculated and difference traces (blue, red and gray, respectively). The blue markers at the bottom indicate the positions of the Bragg's reflection. The peak highlighted by an asterisk in (b) belongs to an impurity. Horizontal axis, $2 \theta$ (deg); vertical axis, intensity (counts). The portion above $40^{\circ}$ has been magnified (4x). 
S.2 Infrared Spectroscopy

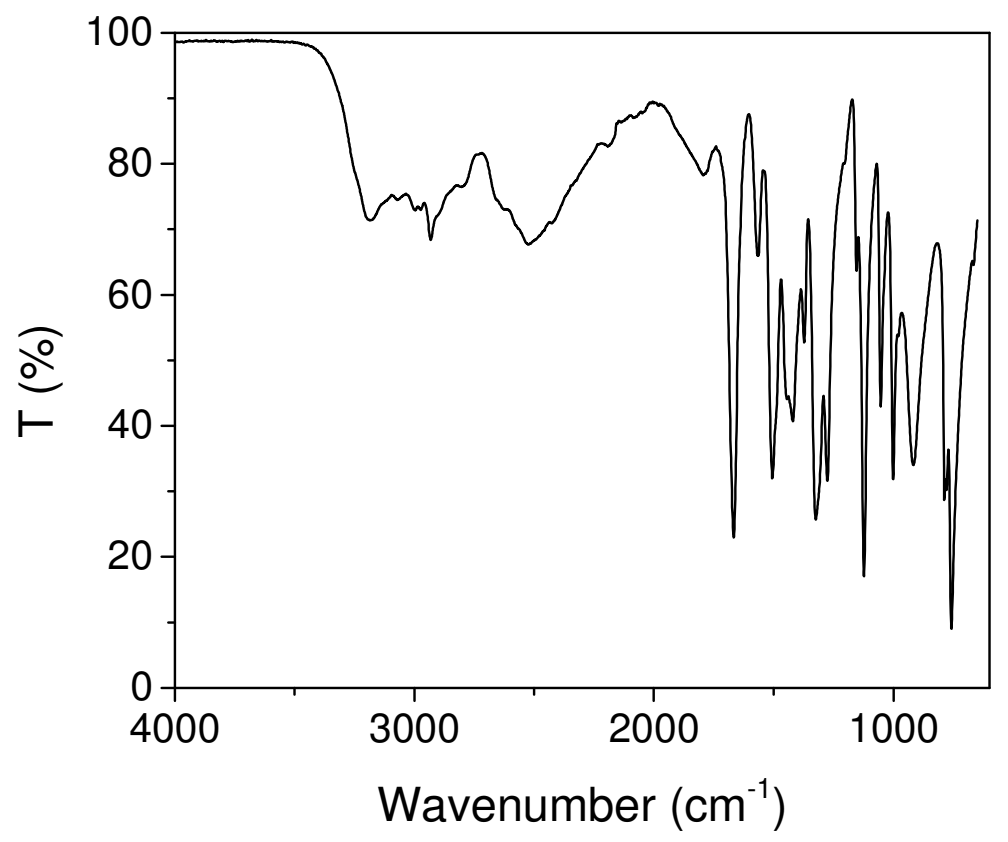

Figure S2. Infrared spectrum of the ligand $\mathrm{H}_{2} \mathrm{dmpzc}$. 


\section{S.3 Thermal Behavior}

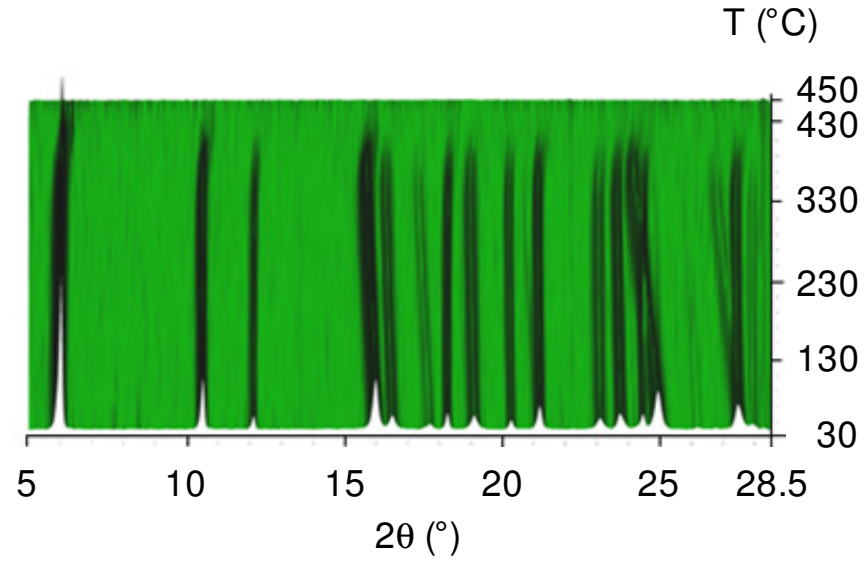

(a)

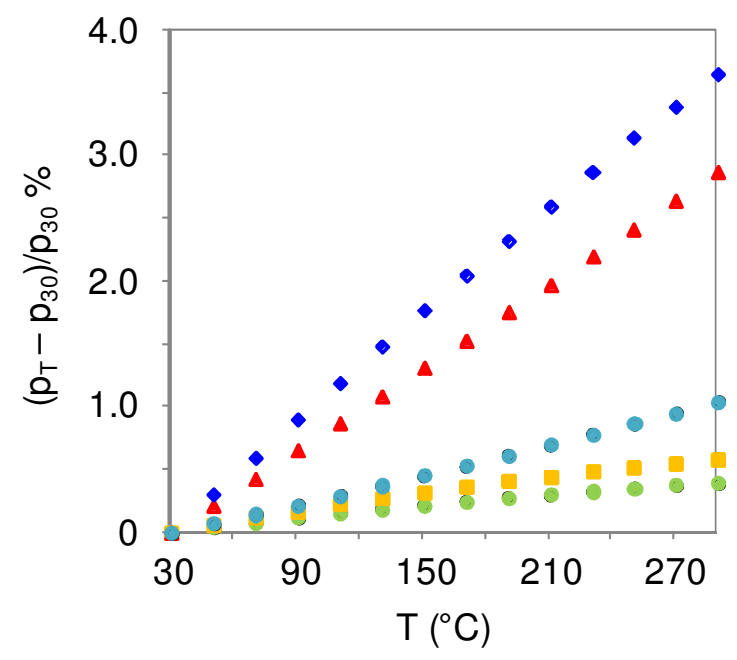

(b)

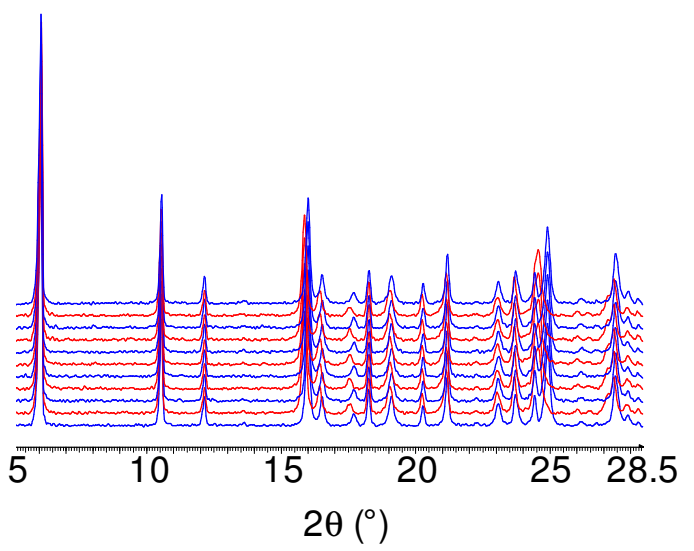

(c)

Figure S3. a) Plot of the powder X-ray diffraction patterns measured on Pd-dmpzc as a function of temperature heating in air, with steps of $20^{\circ} \mathrm{C}$, from $30^{\circ} \mathrm{C}$ up to complete decomposition. b) Percentage variation of the unit cell parameters $\left(\mathrm{p}_{\mathrm{T}}\right)$ of Pd-dmpzc as a function of temperature. At each temperature, the actual values $\left(\mathrm{p}_{\mathrm{T}}\right)$ have been normalized with respect to those at $30{ }^{\circ} \mathrm{C}\left(\mathrm{p}_{30}\right)$. $a$, green circles; $b$, orange squares; $c$, red triangles; $\beta$, cyan circles; $V$, blue rhombi. c) Plot of the powder X-ray diffraction patterns measured on Pd-dmpzc along five heating-cooling cycles in the temperature range $30-150{ }^{\circ} \mathrm{C}$. Heating step, red; cooling step, blue. 


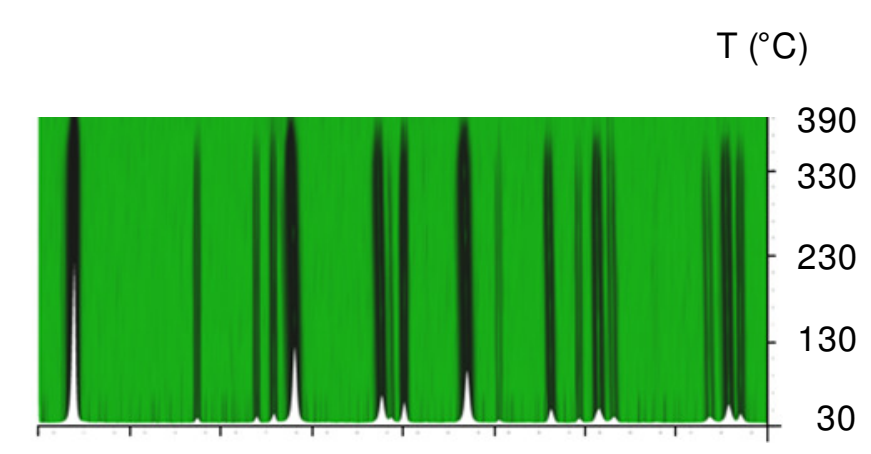

$\begin{array}{lllllllll}9.5 & 12.5 & 15.5 & 18.5 & 21.5 & 24.5 & 27.5 & 30.5 & 33.5\end{array}$

$2 \theta\left({ }^{\circ}\right)$

(a)

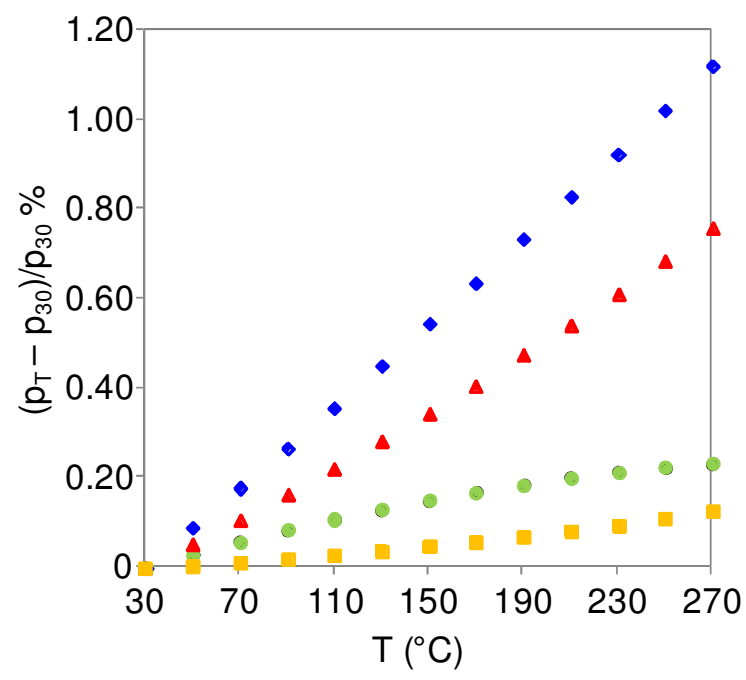

(b)

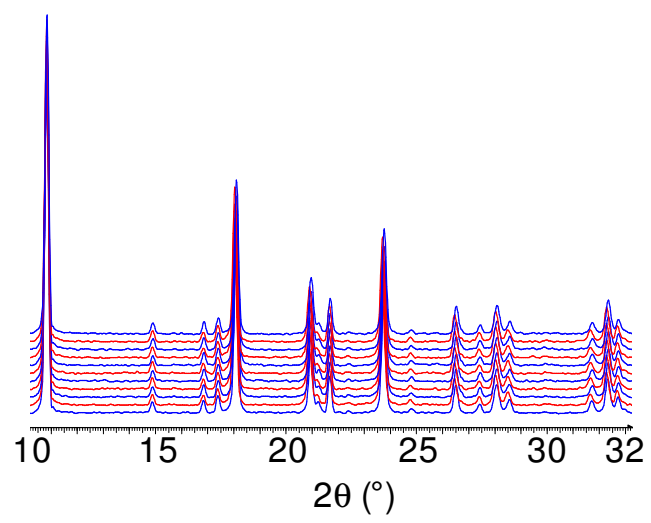

(c)

Figure S4. a) Plot of the powder X-ray diffraction patterns measured on Cu-dmpzc as a function of temperature heating in air, with steps of $20^{\circ} \mathrm{C}$, from $30^{\circ} \mathrm{C}$ up to decomposition. b) Percentage variation of the unit cell parameters $\left(\mathrm{p}_{\mathrm{T}}\right)$ of Cu-dmpzc as a function of temperature. At each temperature, the actual values $\left(\mathrm{p}_{\mathrm{T}}\right)$ have been normalized with respect to those at $30{ }^{\circ} \mathrm{C}\left(\mathrm{p}_{30}\right) . a$, green circles; $b$, orange squares; $c$, red triangles; $V$, blue rhombi. c) Plot of the powder X-ray diffraction patterns measured on Cu-dmpzc along five heating-cooling cycles in the temperature range $30-150{ }^{\circ} \mathrm{C}$. Heating step, cyan; cooling step, blue. 


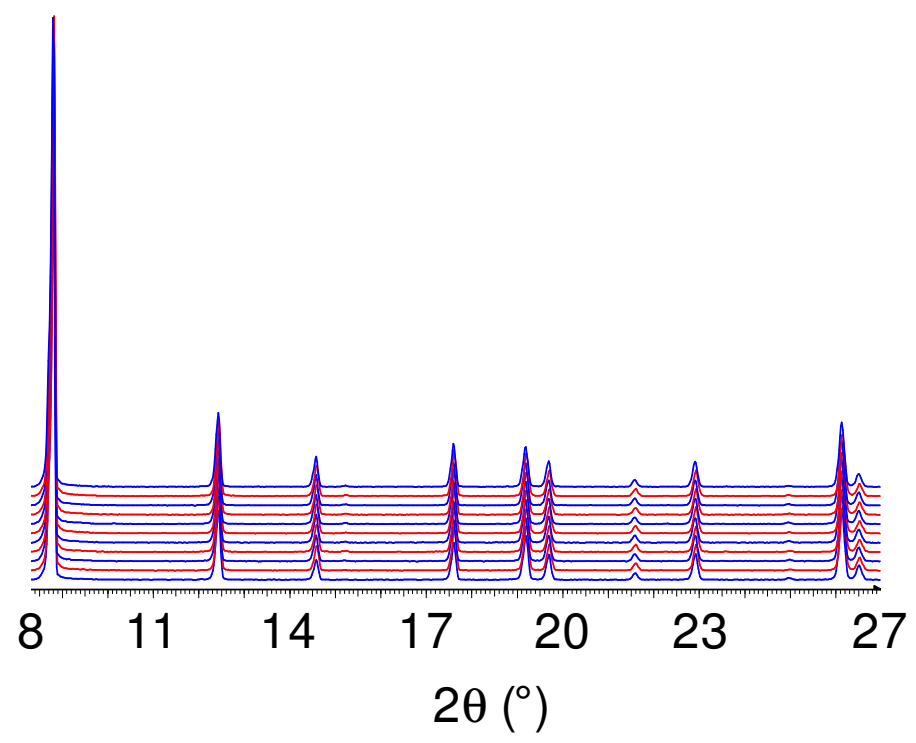

Figure S5. Plot of the powder X-ray diffraction patterns measured on $\mathbf{Z n - d m p z c} \cdot \mathbf{S}$ along five heating-cooling cycles in the temperature range $30-150{ }^{\circ} \mathrm{C}$. Heating step, red; cooling step, blue.
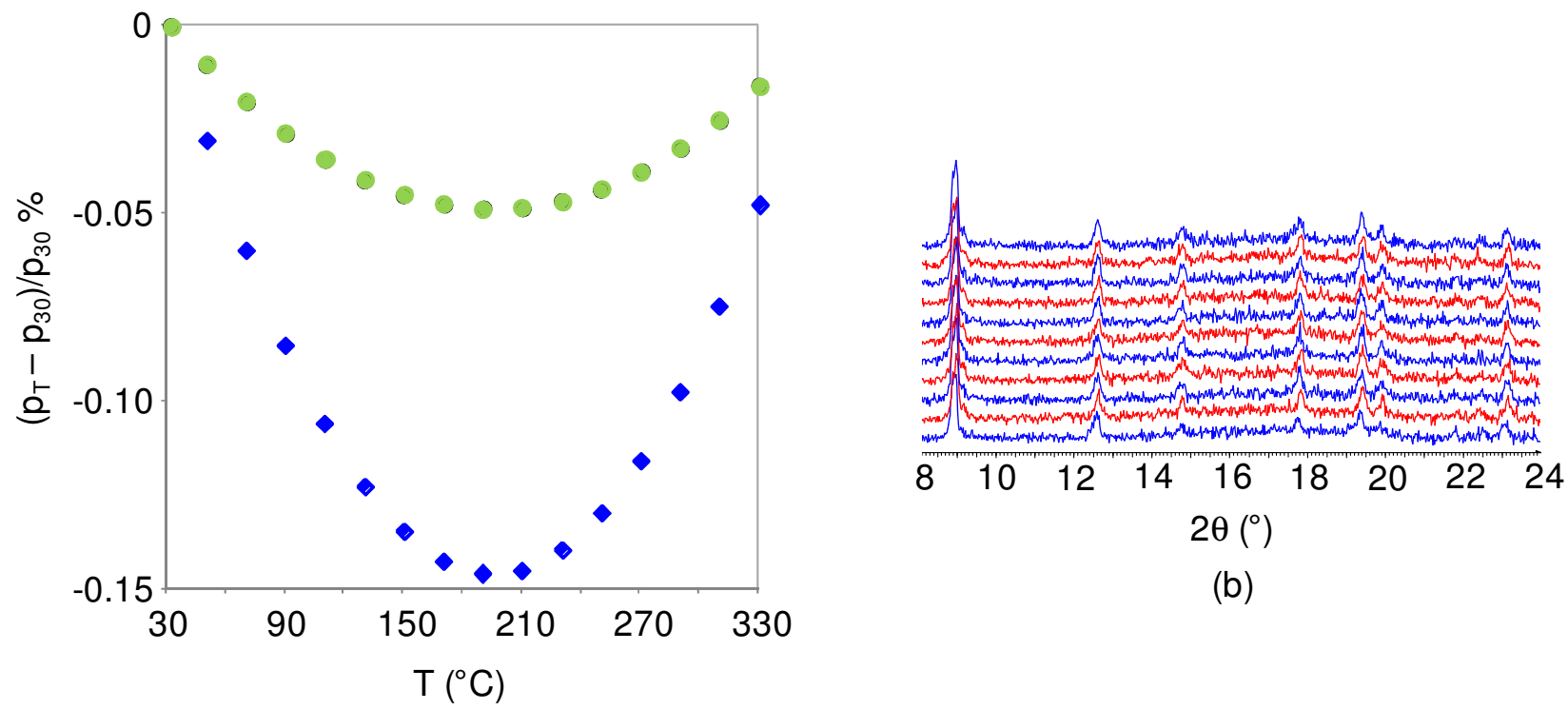

(b)

(a)

Figure S6. a) Percentage variation of the unit cell parameters of $\mathbf{C o - d m p z c} \cdot \mathbf{S}$ as a function of temperature. At each temperature, the actual values $\left(\mathrm{p}_{\mathrm{T}}\right)$ have been normalized with respect to those at $30{ }^{\circ} \mathrm{C}\left(\mathrm{p}_{30}\right) . a$, green circles; $V$, blue rhombi. c) Plot of the powder X-ray diffraction patterns measured on Co-dmpzc·S along five heating-cooling cycles in the temperature range $30-150{ }^{\circ} \mathrm{C}$. Heating step, red; cooling step, blue. 
S.4 Catalytic Activity

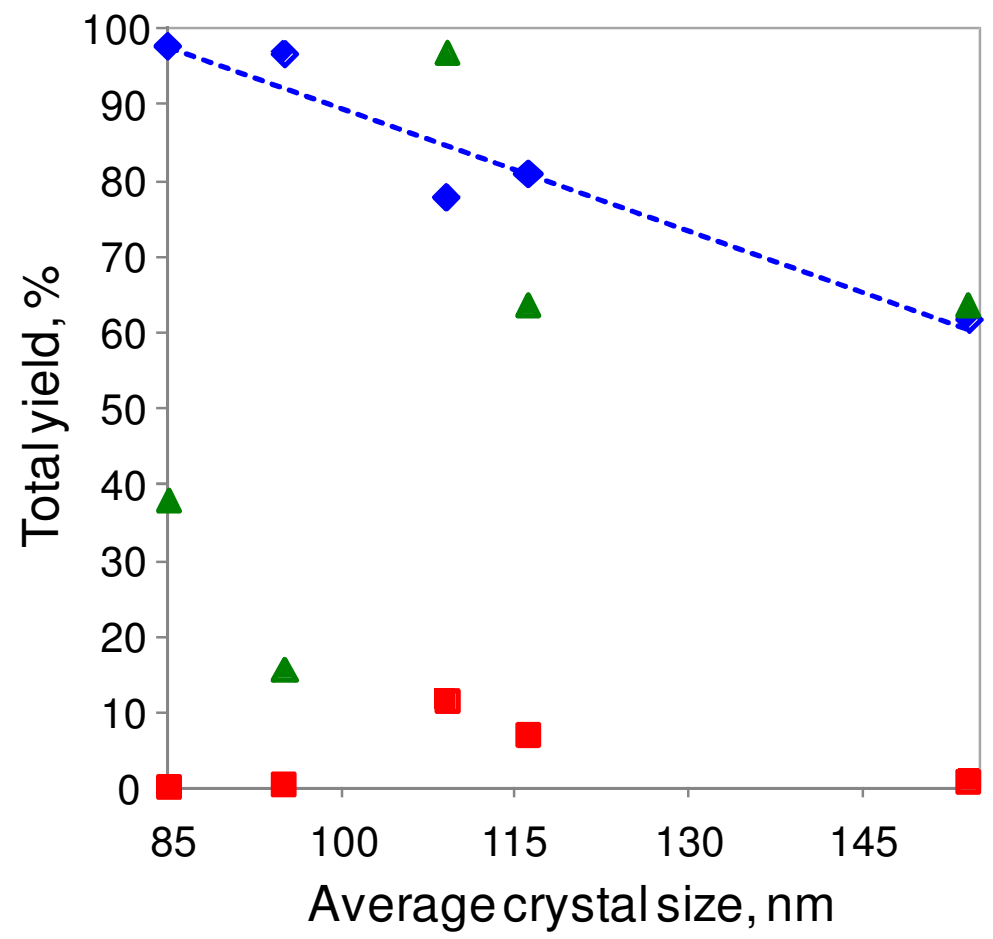

Figure S7: Percentage total yield vs. average crystal size for the oxidation of cyclohexane (red squares; entries 13, 25, 27-29 of Table 1), the oxidation of phenylethanol (green triangles; entries 4, 11, 15, 17, 19 of Table 2), and the Henry C-C coupling reaction (blue rhombi; entries 1, 7, 13, 23, 33 of Table 3). 